\section{Trombosis venosa subclavia y tromboembolismo pulmonar como manifestación de trombopenia inducida por heparina tipo II}

\section{Sr. Director:}

La trombopenia inducida por heparina (HIT) de mecanismo inmune o tipo II es una entidad bien definida, efecto secundario mayor del tratamiento con heparina, tanto no fraccionada (HNF) como de bajo peso molecular (HBPM), cuya incidencia oscila entre el 1 y el $3 \%$ de los tratados, sobre todo con HNF. Su interés principal radica en que con frecuencia es infradiagnosticada pudiendo ser origen de severas complicaciones tromboembólicas y hemorrágicas, con una mortalidad de hasta el $60 \%$. Recientemente atendimos en nuestro hospital un caso presentado en forma de enfermedad tromboembólica como complicación del tratamiento profiláctico con HBPM ,y que se presentó en el postoperatorio tardío de un by-pass gástrico por laparoscopia.

Se trata de una mujer de 38 años sin antecedentes de interés, salvo una obesidad mórbida tratada con by-pass gástrico por laparoscopia en un hospital diferente al nuestro, del que fue alta con Nadroparina cálcica $0,4 \mathrm{mg}$ sc diaria, durante 2 semanas. A los 7 días del tratamiento en domicilio, la paciente comenzó con edema progresivo en brazo izdo, acompañándose 2 días más tarde de intenso dolor torácico en costado y región dorsal derecha de características pleuríticas. A la exploración física destacaba hipoventilación basal derecha así como severo edema con fóvea de todo el miembro superior izquierdo, doloroso a la movilización y con discreta cianosis, con pulsos conservados. En el hemograma se constató: hemoglobina 8,9 g/dL, Hematocrito 27\%, VCM $73 \mathrm{fL}$, leucocitos $10.600 / \mathrm{mm}^{3}$, plaquetas $92.000 / \mathrm{mm}^{3}$. La bioquímica era anodina, y en la coagulación presentaba TP(ratio) 1,1, PTTA (ratio) 0,8, Fibrinógeno $546 \mathrm{mg} / \mathrm{dl}$ y dímero D $2.997 \mathrm{ng} / \mathrm{mL}$. En la placa de tórax se apreció elevación de hemidiafragma derecho con condensación basal derecha y borramiento del seno costofrénico homolateral. Se practicó un ecocardiograma, que fue normal salvo ligera elevación de la presión sistólica en arteria pulmonar, y un TAC torácico, mostrando infiltrado basal derecho con reacción pleural y sin broncograma visible. En la eco-doppler del miembro superior izquierdo aparecían signos de trombosis en venas sub- clavia, axilar y humeral. La gammagrafía pulmonar de ventilación-perfusión mostró un patrón de perfusión pulmonar de intermedia-alta probabilidad de TEP. Al ingreso en Urgencias se inició perfusión de heparina sódica IV, no obteniéndose buenos tiempos de anticoagulación ni respuesta clínica a las 24 horas del inicio del tratamiento. Una vez en planta y tras diversos ajustes de la dosificación, continuaba sin respuesta ni niveles terapéuticos, con progresiva disminución del número de plaquetas. Ante estos hallazgos se interpreta como una posible trombocitopenia asociada a heparina tipo II, ya que se considera de aparición tardía al haber estado tratada previamente en su domicilio con HBPM y ser las plaquetas a su ingreso de 90.000. Por ello se suspendió la heparina $\mathrm{Na}$ y se inició tratamiento con Lepirudina, que consiguió buenos niveles de coagulación iniciando posteriormente acenocumarol.

La HIT tipo II consiste en una reducción del recuento plaquetario mayor del $50 \%$ que ocurre generalmente entre 4 y 14 dias tras la exposición a heparina, aunque el inicio puede ser más precoz, incluso en horas, en caso de reexposición (sobre todo los 100 dias anteriores). Fisiopatológicamente se conoce que la heparina se une al factor 4 plaquetario formando un hapteno fuertemente inmunógeno, inductor de la producción de anticuerpos específicos de tipo Ig G. Consecuencia de dicha interacción tiene lugar la activación plaquetaria, con posterior lisis y aglutinación. A ello se le une la activación por parte de dichos anticuerpos de monocitos y células endoteliales, con incremento en los niveles de Factor Tisular, contribuyendo al estado procoagulante. A pesar de que pueden aparecer manifestaciones hemorrágicas, en general poco frecuentes aunque potencialmente graves, suelen presentarse como complicaciones tromboembólicas $(30-40 \%)$ tanto venosas como arteriales, precisando amputación de extremidades hasta en un $5 \%$ de casos. El tromboembolismo venoso se da con mayor frecuencia en extremidades inferiores, pudiendo aparecer no obstante como TEP, en adrenales, cerebro o miembros superiores. También se ha descrito la aparición de CID en el 5-10\% de los casos. Su diagnóstico debe basarse en evidencias clínicas y de laboratorio; se recomienda realizar recuentos plaquetarios dos veces entre el cuarto y el décimo día de tratamiento, aunque en casos de reexposición se iniciará en las primeras 24 horas. Existen pruebas funcionales, que miden el grado de activación plaquetaria, e inmunológicas, que pueden ayudar al diagnóstico. Ante su sospecha está indicada la suspensión inmediata del tratamiento, e inicio de anticoagulación alternativa. Nunca se deben emplear antivitaminas $\mathrm{K}$ aisladas (riesgo de necrosis cutánea), filtros de vena cava ni transfusiones de plaquetas. Entre los inhibidores directos de la trombina disponibles, Danaparinoid, Argatroban, Fondaparinux y Lepirudina, ésta última es el único fármaco aprobado en España para esta indicación. Se trata de una hirudina recombinante de excreción renal, capaz de inducir anticuerpos anti-hirudina, que en casos aislados han ocasionado muerte por shock anafiláctico, y frente al cual no se dispone de antídoto.

Es pues importante tener siempre presente que ante una complicación trombótica de un paciente tratado previamente con Heparina, debemos descartar una disminución del recuento plaquetario $>50 \%$ con respecto a la cifra basal, y si esto existe suspenderla inmediatamente y utilizar tratamientos anticoagulantes alternativos. Mientras, se deberia intentar confirmar el diagnóstico con pruebas funcionales y detección de anticuerpos específicos en los hospitales que dispongan de estos medios.

\section{B. Alcaraz Vidal, G. García Parra, P. J. García López,} M. Artero Castro, A. Pérez Gracia

Servicio de Medicina Interna. Hospital Santa María del Rosell. Cartagena. Murcia 
1. Warkentin TE, Greinacher A. Heparin-Induced thrombocytopenia: recognition, treatment and prevention. The Seventh ACCP Conference on Antithrombotic and Thrombolytic Therapy. Chest 2004; 126: 311S-337S.

2. Van Der Bemt P, Meyboom R, Egberts A. Drug-Induced Immune Thrombocytopenia. Drug Safety 2004; 27: 12431252.

3. Hirsh J, Heddle N, Kelton JG. Treatment of Heparin-Induced Thrombocytopenia. Arch Inter Med 2004; 164: 361-369.

4. Warkentin TE. Heparin-Induced Thrombocytopenia. Diagnosis and management. Circulation 2004; 110: e454e458. 